\title{
Is the massive star cluster Westerlund 2 double? - A high resolution multi-band survey with the Hubble Space Telescope
}

\author{
Peter Zeidler ${ }^{1,2}$, Antonella Nota ${ }^{2,3}$, Elena Sabbi ${ }^{2}$, Eva K. Grebel ${ }^{1}$, \\ Monica Tosi ${ }^{5}$, Alceste Z. Bonanos ${ }^{4}$, Anna Pasquali ${ }^{1}$, Carol Christian ${ }^{2}$ \\ and Selma E. de Mink ${ }^{6}$ \\ ${ }^{1}$ Astronomisches Rechen-Institut, Zentrum für Astronomie der Universität Heidelberg, \\ Mönchhofstr. 12-14, 69120 Heidelberg, Germany \\ email: pzeidler@ari.uni-heidelberg.de \\ ${ }^{2}$ Space Telescope Science Institute, 3700 San Martin Drive, Baltimore, MD 21218, USA \\ ${ }^{3}$ ESA, SRE Operations Devision \\ ${ }^{4}$ IAASARS, National Observatory of Athens, GR-15326 Penteli, Greece \\ ${ }^{5}$ INAF - Osservatorio Astronomico di Bologna \\ ${ }^{6}$ Astronomical Institute Anton Pannekoek, Amsterdam University, Science Park 904, 1098 XH, \\ Amsterdam, The Netherlands
}

\begin{abstract}
Westerlund $2(\mathrm{Wd} 2)$ is one of the most massive young star clusters known in the Milky Way. The close proximity $(4.16 \mathrm{kpc})$ to the Sun, and the young age $(2.0 \mathrm{Myr})$ allow us to study star formation in detail at a high spatial resolution. We present results from our recent deep multi-band survey in the optical and near-infrared obtained with the Hubble Space Telescope. We demonstrated that, as expected, the region is affected by significant differential reddening with a median value of $E(B-V)_{g}=1.87 \mathrm{mag}$. The distance was inferred from the dereddened color-magnitude diagrams using Padova isochrones. Analyzing the spatial distribution of stars we found that Wd2 consists of two sub-clumps, namely the main cluster of Westerlund 2 and a less well populated clump located to the North. We estimated the same age of 0.1-2.0 Myr for both sub-clumps, thus we conclude that they are likely coeval.
\end{abstract}

Keywords. techniques: photometric - stars: early type - stars: pre-main sequence - HII regions - open clusters and associations: individual (Westerlund 2) - infrared: stars

\section{Introduction}

Westerlund 2 (hereafter $\mathrm{Wd} 2$; Westerlund 1961) is one of the most massive $(M \geqslant$ $10^{4} M_{\odot}$, Ascenso et al. 2007) young (0.1-2.0 Myr) star clusters in the Milky Way (MW). It is located in the Carina-Sagittarius arm $(\alpha, \delta)=\left(10^{h} 23^{m} 58^{s} .1,-57^{\circ} 45^{\prime} 49^{\prime \prime}\right)$ (J2000) at a distance of $\sim 4.16 \mathrm{kpc}$ from the Sun (Zeidler et al. 2015a). It is the central ionizing cluster of the giant HII cloud RCW 49 (Rodgers et al. 1960). The estimated molecular cloud mass ranges from $(1.7 \pm 0.8-7.5) \times 10^{5} \mathrm{M}_{\odot}$ (Furukawa et al. 2009; Dame 2007, both estimates based on CO submillimeter/milimeter spectroscopy). Moffat et al. (1991) estimated that Wd2 hosts more than 80 O-type stars. Due to its close proximity to the Sun and its young age, $\mathrm{Wd} 2$ is a perfect target to study young massive star clusters very close to their earliest stages.

We are currently leading a high resolution, multi-band study of $\mathrm{Wd} 2$ with the $H u b$ ble Space Telescope (HST) (PI: A. Nota). We observed Wd2 in four wide-band filters, namely $F 555 W, F 814 W, F 125 W$, and $F 160 W$, and two narrow-band filters $(F 658 N$ and $F 128 N$ ) centered on the $\mathrm{H} \alpha$ and $\mathrm{Pa} \beta$ line emission. The optical observations were acquired with the Advanced Camera for Surveys (ACS, Ubeda et al. 2012) and the 
infrared (IR) observations were obtained with the Wide Field Camera 3 in the IR channel (WFC3/IR, Dressel 2012). The field of view (FOV) is $\sim 16-20 \mathrm{arcmin}^{2}$. Our final photometric catalog comprises 17,121 objects. 2236 point sources are detected in all six filters. The limiting magnitudes are $F 555 W=26.7 \mathrm{mag}, F 814 W=25.3 \mathrm{mag}$, $F 125 W=23.1 \mathrm{mag}$, and $F 160 W=22.6 \mathrm{mag}$. Our optical data are $\sim 5$ mag deeper than the photometric data used by Vargas Álvarez et al. (2013) obtained with the Wide-Filed Planetary Camera 2 (WFPC2) (Gonzaga 2010). The near-infrared data reach 3-5 mag deeper than those of Ascenso et al. (2007). A detailed description of the observations, the data reduction, and the photometric catalog is presented in Zeidler et al. (2015a). Our observations were chosen as the $25^{\text {th }}$ anniversary picture for the HST (see Fig. 1).

Our goals are a full census of the stellar population in $\mathrm{Wd} 2$ down to $0.1 M_{\odot}$, its spatial distribution, its pre-main-sequence (PMS) component, its mass function, and the total cluster and stellar mass. Additionally, we will see if $\mathrm{Wd} 2$ already undergoes mass segregation, what is already seen at massive star clusters of this age (e.g. NGC 3603, Pang et al. 2013).

\section{The gas excess toward $\mathrm{Wd} 2$}

Wd2 is the central cluster of the giant HII region RCW 49. Due to its location in the Galactic disk it suffers from high differential reddening. Using the $\mathrm{H} \alpha$ and $\mathrm{Pa} \beta$ line emission we created a high resolution, pixel-to-pixel map of the gas excess $E(B-V)_{g}$. This map gives us the capability of individually deredden the cluster stars assuming that the gas and dust are located between us and $\mathrm{Wd} 2$.

\subsection{The pixel-to-pixel gas excess map}

In order to create the color excess map, we removed the continuum emission pixel by pixel. Therefore, we linearly extrapolated the continuum flux at the position of $F 658 \mathrm{~N}$ $(F 128 N)$ using the $F 555 W$ and $F 814 W(F 125 W$ and $F 160 W)$ observations. Calzetti et al. (1996) provide a relation between the gas excess $E(B-V)_{g}$, the observed $\left(R_{o b s}\right)$, and theoretical flux ratio $\left(R_{t h}\right)$ of $\mathrm{H} \alpha$ and $\mathrm{Pa} \beta$ :

$$
E(B-V)=\frac{-\log \left(R_{o b s} / R_{\text {int }}\right)}{0.4[\kappa(\mathrm{H} \alpha)-\kappa(\mathrm{Pa} \beta)]} .
$$

For the theoretical flux ratio $R_{\text {int }}$ we derived a value of 17.546 with an assumed electron temperature of $10000 \mathrm{~K}$ and an electron density of $100 \mathrm{~cm}^{-3}$ (Osterbrock \& Bochkarev 1989). We adopted a normal total-to-selective extinction of $R_{V}=3.1$. Using the extinction law of Cardelli et al. (1989) we derived $\kappa(\mathrm{H} \alpha)=2.437$ and $\kappa(\mathrm{Pa} \beta)=0.8035$.

The median $E(B-V)_{g}$ is $1.87 \mathrm{mag}$ with a median uncertainty of $\sigma_{E(B-V)}=0.032 \mathrm{mag}$, which is of the order of our photometric uncertainties.

\subsection{Transformation of the gas excess to a stellar excess}

To use the color-excess map for our stellar photometry we needed to transform the gas excess into a stellar excess. Calzetti et al. (2000) showed a linear relation between the gas excess $E(B-V)_{g}$ and the stellar excess $E(B-V)_{\star}$. Up to now 36 OB-stars were spectroscopically observed in Wd2 (Rauw et al. 2004, 2007, 2011; Vargas Álvarez et al. 2013). Comparing the theoretical colors (Sparke \& Gallagher 2007) observed colors of the stars with spectral type, we can infer the color excess $E(B-V)$. The median values of the gas excess transforms to a stellar excess of $E(B-V)_{\star}=1.55$ mag. This procedure is described in detail in Zeidler et al. (2015a). 


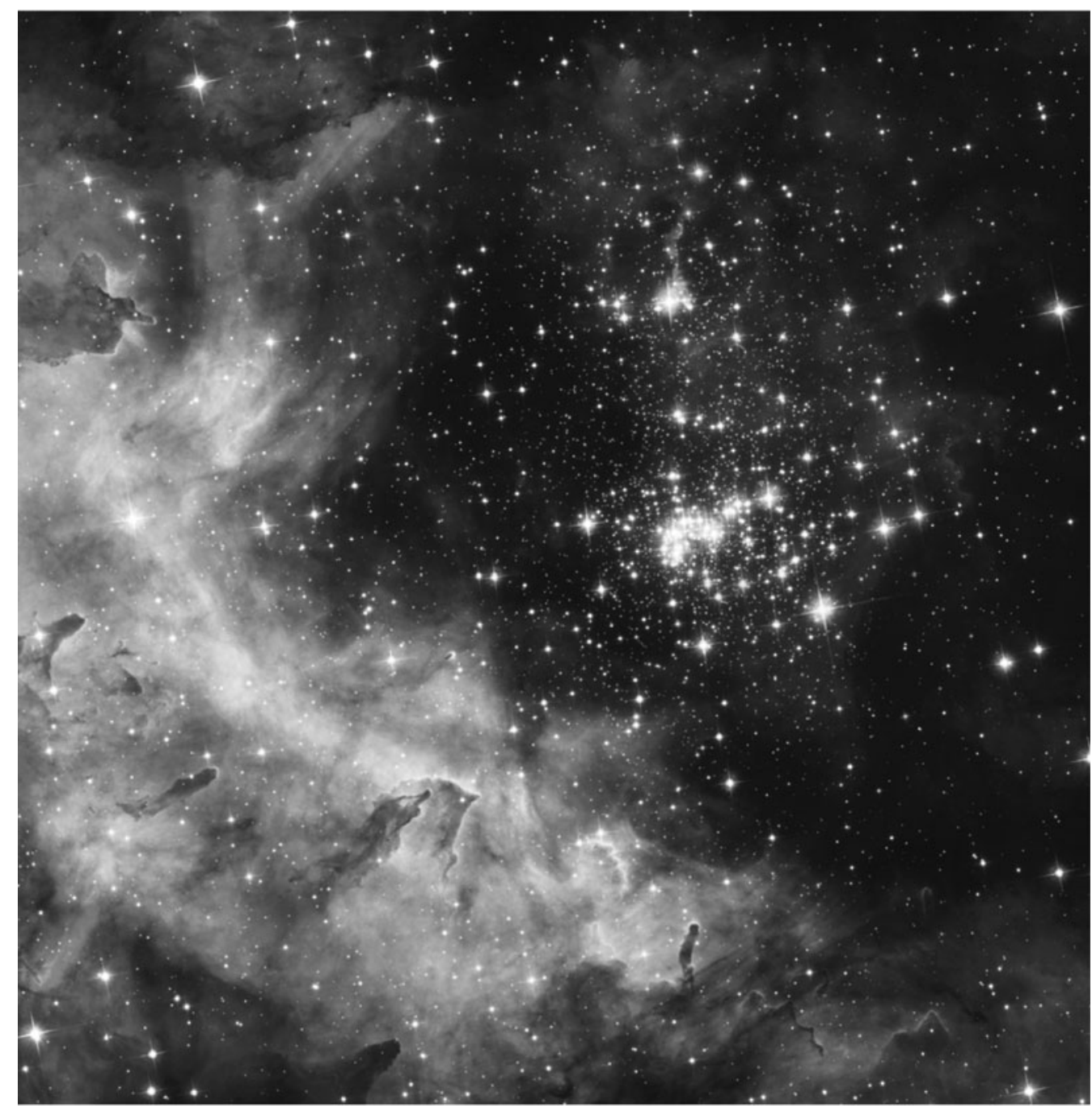

Figure 1. Color composite image of the HST ACS and WFC3/IR data of Wd2, including the $F 125 W$ (red), $F 814 W$ (green), and $F 555 W$ (blue) filters (a color version is published in Zeidler et al. (2015a)). North is up, East to the left. The FOV is $\sim 4 \operatorname{arcmin} \times 4$ arcmin. This image was chosen to be the official Hubble 25th anniversary image. Credit: NASA, ESA, the Hubble Heritage Team (STScI/AURA), A. Nota (ESA/STScI), and the Westerlund 2 Science Team.

\section{The cluster population}

\subsection{The separation of the cluster members}

Due to its location in the Galactic disk, the $\mathrm{Wd} 2$ cluster population is contaminated by a high number of foreground objects. In order to get a proper census of the the stellar content in $\mathrm{Wd} 2$, as well as its spatial distribution we had to separate the cluster members from the foreground stars. Plotting the $F 814 W-F 160 W$ vs. $F 814 W$ color-magnitude diagram (CMD) of our catalog (see left frame of Fig. 2), we see two populations, a redder cluster member population and a bluer foreground population. The clear gap in their colors gives us the opportunity to separate the two populations with an arbitrary straight line. To show that the blue sequence is foreground, we simulated the MW disk 

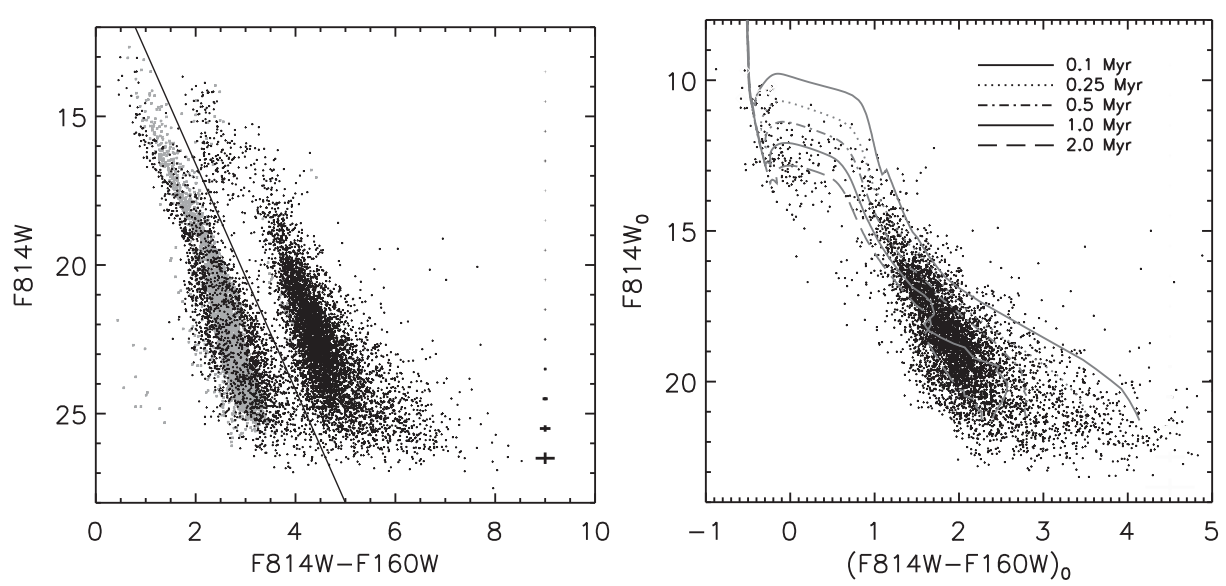

Figure 2. Left: The $F 814 W$ vs. $(F 814 W-F 160 W)$ CMD of the 7,697 point sources from the $\mathrm{Wd} 2$ photometric catalog. The points right to the straight line represent likely members of the RCW 49 region (including cluster members). The left sources are probable foreground stars. The gray points indicate the location of the synthetic objects from the Besançon model in the direction of $\mathrm{Wd} 2$. The black solid line marks our arbitrary separation between cluster and field stars. Right: The dereddened $F 814 W_{0}$ vs. $(F 814 W-F 160 W)_{0}$ CMD. Overlayed are the PARSEC 2.1S (Bressan et al. 2012) isochrones between 0.1 and $2.0 \mathrm{Myr}$ for $Z=Z_{\odot}$. The diamonds mark the stars with known spectral type.

with the Besançon model (Robin et al. 2003) and indeed the gray points in the left-hand plot of Fig. 2 (representing the model) fall onto the blue sequence.

\subsection{The dereddened $C M D$ of $W d 2$}

After the selection of likely cluster members, we used our high resolution gas excess map to deredden the photometry of each star. The total-to-selective extinction of $R_{V}=3.95$ was determined via distance independent two-color diagrams (TCD, Zeidler et al. 2015a). In the right panel of Fig. 2 one can see the dereddened, cluster members in the $F 814 W_{0}$ vs. $(F 814 W-F 160 W)_{0}$ CMD with overplotted Padova PARSEC 1.2S (Bressan et al. 2012) isochrones in the range of $0.1-2.0 \mathrm{Myr}$ for solar metallicity $Z=0.0153$. The isochrones extend down to a stellar mass of $0.1 M_{\odot}$ Using the turn-on (TO) region we determined a distance for Wd2 of $4.16 \mathrm{kpc}$ in good agreement with Vargas Álvarez et al. (2013). The isochrones give an upper age limit of $\sim 2$ Myr for $\mathrm{Wd} 2$.

\section{The spatial distribution of the stellar content}

After selecting the $\mathrm{Wd} 2$ members we analyzed their spatial distribution. In order to compensate for incompleteness effects, we made magnitude cuts, using the luminosity function, where our observations can be considered as mostly complete. We used all objects with $F 814 W<22.75 \mathrm{mag}$ and $F 160 W<18.00 \mathrm{mag}$. This leaves us with 2610 point sources. We created a $2 \mathrm{D}$ density map of the stars (see left-hand plot of Fig. 3) calculating the local surface density within a radius of $r<10.8^{\prime \prime}$ from each star. This radius ensures, that we always have enough objects per density bin, yet we are not losing any small scale structures.

We fitted a combination of two 2D Gaussians with a common offset to the smoothed (binsize: 4 px) density map with independently determined peak positions and maximums of the two appearing sub-clumps. We took the 1- $\sigma$ limits of the two peaks to define the two sub-clumps (thick black contours in Fig. 3), namely the main cluster (MC) and the 

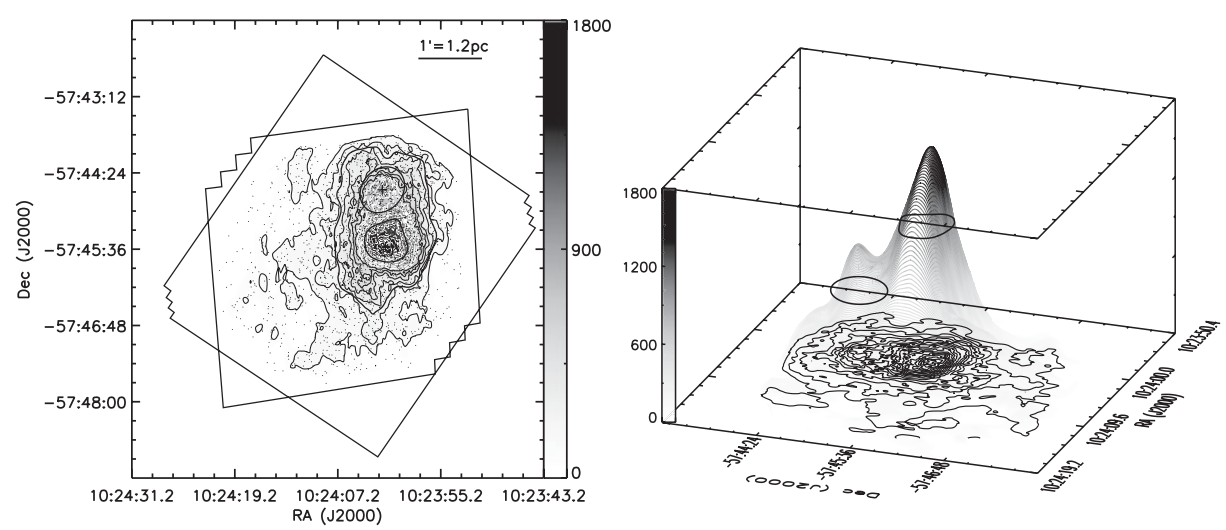

Figure 3. Left: Using the 2610 point sources selected with $F 814 W<22.75$ mag and $F 160 W<18.00 \mathrm{mag}$, we created a 2D plot of the stellar density, taking into account all objects within a radius of $r<10.8^{\prime \prime}$ around each star. The thick inner contours mark the two subclumps, namely the main cluster and the northern clump. The thick outer contour separates the $\mathrm{Wd} 2$ cluster from the periphery. The color-bar indicates the spatial density in $\operatorname{arcmin}^{-2}$ Right: $3 \mathrm{D}$ representation of the smoothed spatial distribution. The z-axis represents the density in $\operatorname{arcmin}^{-2}$.

northern clump (NC). We separated the cluster of $\mathrm{Wd} 2$ from the peripheral region of RCW 49 using the $2-\sigma$ contour line (outer thick black contour in Fig. 3 ). In the right-hand plot of Fig. 3 one can see the $3 \mathrm{D}$ representation of the smoothed stellar distribution. The peak coordinates for the MC are R.A. $=10^{\mathrm{h}} 24^{\mathrm{m}} 01^{\mathrm{s}} .65$ and Decl. $=-57^{\circ} 45^{\prime} 34^{\prime \prime} 0$. and for the $\mathrm{NC}$ we got R.A. $=10^{\mathrm{h}} 24^{\mathrm{m}} 02^{\mathrm{s}} .01$ and Decl. $=-57^{\circ} 44^{\prime} 39^{\prime \prime} 2$. . This leads to a distance of the two sub-clumps of $d=54.2^{\prime \prime}(1.08 \mathrm{pc})$. The areas of the MC and the NC cover $0.34 \operatorname{arcmin}^{2}\left(0.49 \mathrm{pc}^{2}\right)$ and $0.39 \operatorname{arcmin}^{2}\left(0.56 \mathrm{pc}^{2}\right)$, respectively.

The two sub-clumps have the same projected size, but the spatial density of the main cluster is more than a factor two higher. These radii are comparable to the core radii found by Kuhn et al. (2014) for 17 cluster regions. The individual CMDs show the same age for both sub-clumps. This leads to the conclusion that both sub-clumps are coeval. For a detailed analysis we refer to Zeidler et al. (2015a) and Zeidler et al. (2015b).

\section{Summary and Conclusions}

We presented a detailed analysis of the stellar content of Wd2 using high resolution multi-band observations with the HST. Combing the $\mathrm{H} \alpha$ and $\mathrm{Pa} \beta$ line emissions we created a high-resolution, pixel-to-pixel $E(B-V)_{g}$ gas excess map. Using the catalog of stars with spectral types, we managed to transform this gas excess to a stellar excess $E(B-V)_{\star}$. With this we were able to deredden the photometry of the stars in $\mathrm{Wd} 2$. To purge our catalog of foreground objects we used the $F 814 W$ vs. ( $F 814 W-F 160 W$ ) CMD. Two populations, clearly separated by $\sim 1$ mag (see left-hand plot of Fig. 2 ) are visible.

Using PARSEC 1.2S (Bressan et al. 2012) isochrones and plotting them over the dereddened cluster CMD (see right-hand plot of Fig. 2) we could infer an age of 0.1-2 Myr and a distance of $4.16 \mathrm{kpc}$ for $\mathrm{Wd} 2$. In conclusion, $\mathrm{Wd} 2$ is of the same age or even younger than other very young star clusters like NGC 3603 (Pang et al. 2013) or R136 in the Large Magellanic Cloud (Walborn \& Blades 1997; Sabbi et al. 2012). It is also younger than Westerlund 1, the most massive known young star cluster in the MW (Clark et al. 2005; Gennaro et al. 2011; Lim et al. 2013). 
Fitting two 2D Gaussians to the spatial distributions of cluster members (see Fig. 3) we revealed that $\mathrm{Wd} 2$ is composed of two coeval sub-clumps, the $\mathrm{MC}$ and the $\mathrm{NC}$ having roughly the same spatial extent. The NC contains less than half of the number of objects than the MC. Over-plotting isochrones over the sub-clumps shows that both sub-clumps have the same age of 0.1-2 Myr. Sub-clustering is very common in young clusters (e.g. Kuhn et al. 2014). The radii of the sub-clumps are of the same order as the sub-clusters found by Kuhn et al. (2014) in 17 star forming regions.

\section{References}

Ascenso, J., Alves, J., Beletsky, Y., \& Lago, M. T. V. T. 2007, A\&A, 466, 137

Bressan, A., Marigo, P., Girardi, L., et al. 2012, MNRAS, 427, 127

Calzetti, D., Armus, L., Bohlin, R. C., et al. 2000, ApJ, 533, 682

Calzetti, D., Kinney, A. L., \& Storchi-Bergmann, T. 1996, ApJ, 458, 132

Cardelli, J. A., Clayton, G. C., \& Mathis, J. S. 1989, ApJ, 345, 245

Clark, J. S., Negueruela, I., Crowther, P. A., \& Goodwin, S. P. 2005, A\&A, 434, 949

Dame, T. M. 2007, ApJ, 665, L163

Dressel, L. 2012, Wide Field Camera 3 Instrument Handbook for Cycle 21 v. 5.0 (Baltimore: STScI)

Furukawa, N., Dawson, J. R., Ohama, A., et al. 2009, ApJ, 696, L115

Gennaro, M., Brandner, W., Stolte, A., \& Henning, T. 2011, MNRAS, 412, 2469

\&Gonzaga, S.and Biretta, J. 2010, in HST WFPC2 Data Handbook, v. 5.0 ,ed., Baltimore, STScI (Baltimore: STScI)

Kuhn, M. A., Feigelson, E. D., Getman, K. V., et al. 2014, ApJ, 787, 107

Lim, B., Chun, M.-Y., Sung, H., et al. 2013, AJ, 145, 46

Moffat, A. F. J., Shara, M. M., \& Potter, M. 1991, AJ, 102, 642

Osterbrock, D. E. \& Bochkarev, N. G. 1989, Soviet Ast., 33, 694

Pang, X., Grebel, E. K., Allison, R. J., et al. 2013, ApJ, 764, 73

Rauw, G., Manfroid, J., Gosset, E., et al. 2007, A\&A, 463, 981

Rauw, G., Sana, H., \& Nazé, Y. 2011, A\&A, 535, A40

Rauw, G., De Becker, M., Nazé, Y., et al. 2004, A\&A, 420, L9

Robin, A. C., Reylé, C., Derrière, S., \& Picaud, S. 2003, A\&A, 409, 523

Rodgers, A. W., Campbell, C. T., \& Whiteoak, J. B. 1960, MNRAS, 121, 103

Sabbi, E., Lennon, D. J., Gieles, M., et al. 2012, ApJ, 754, L37

Sparke, L. S. \& Gallagher, III, J. S. 2007, Galaxies in the Universe: An Introduction (Cambridge University Press)

Ubeda et al. 2012, Advanced Camera for Surveys Instrument Handbook for Cycle 21 v. 12.0 (Baltimore: STScI)

Vargas Álvarez, C. A., Kobulnicky, H. A., Bradley, D. R., et al. 2013, AJ, 145, 125

Walborn, N. R. \& Blades, J. C. 1997, ApJS, 112, 457

Westerlund, B. 1961, Arkiv for Astronomi, 2, 419

Zeidler, P., Sabbi, E., Nota, A., et al. 2015a, AJ, 150, 78

Zeidler, P., Nota, A., Sabbi, E., et al. 2015b, in prep. 